\title{
Full-blown hyperparathyroidism
}

\author{
Woo Kyun Bae and Ik-Joo Chung
}

Department of Internal Medicine, Chonnam National University Medical School, Gwangju, Korea

Received: June 4, 2012

Revised : February 14, 2017

Accepted: August 18, 2017

\section{Correspondence to}

Woo Kyun Bae, M.D.

Tel: +82-61-379-7623

Fax: +82-61-379-8019

E-mail: drwookyun@jnu.ac.kr
A 32-year-old man presented to the emergency department with a history of dyspnea, constipation, abdominal pain, and left-arm pain. His past history was significant for uncontrolled parathyroid carcinoma for 9 years. Radiographic findings included fracture of the left humerus, extensive osteolytic lesions of the humeri (arrowheads), calcification in the pancreas (black arrows), nephrolithiasis (white arrows), brown tumor in the right 8th rib, hypercalcemic cardiomyopathy, and rugger-jersey spine (Figs. 1 and 2). The results of the initial laboratory tests were as follows: serum calcium $14.3 \mathrm{mg} / \mathrm{dL}$ (normal range, 8.4 to 10.2); ionized calcium $3.92 \mathrm{mEq} / \mathrm{L}$ (normal range, 2.2 to 2.6); and serum parathyroid hormone

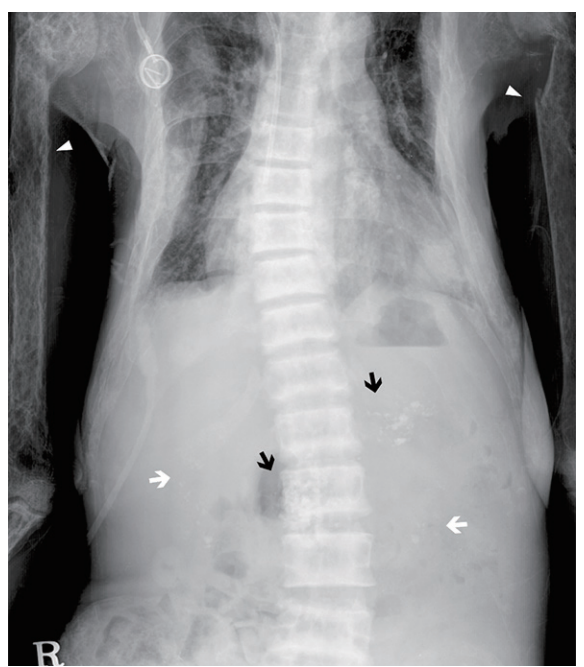

Figure 1. Radiograph shows fracture of the left humerus, extensive osteolytic lesions of the humeri (arrowheads), calcification in the pancreas (black arrows), nephrolithiasis (white arrows), brown tumor in the right 8 th rib, hypercalcemic cardiomyopathy, and rugger-jersey spine.
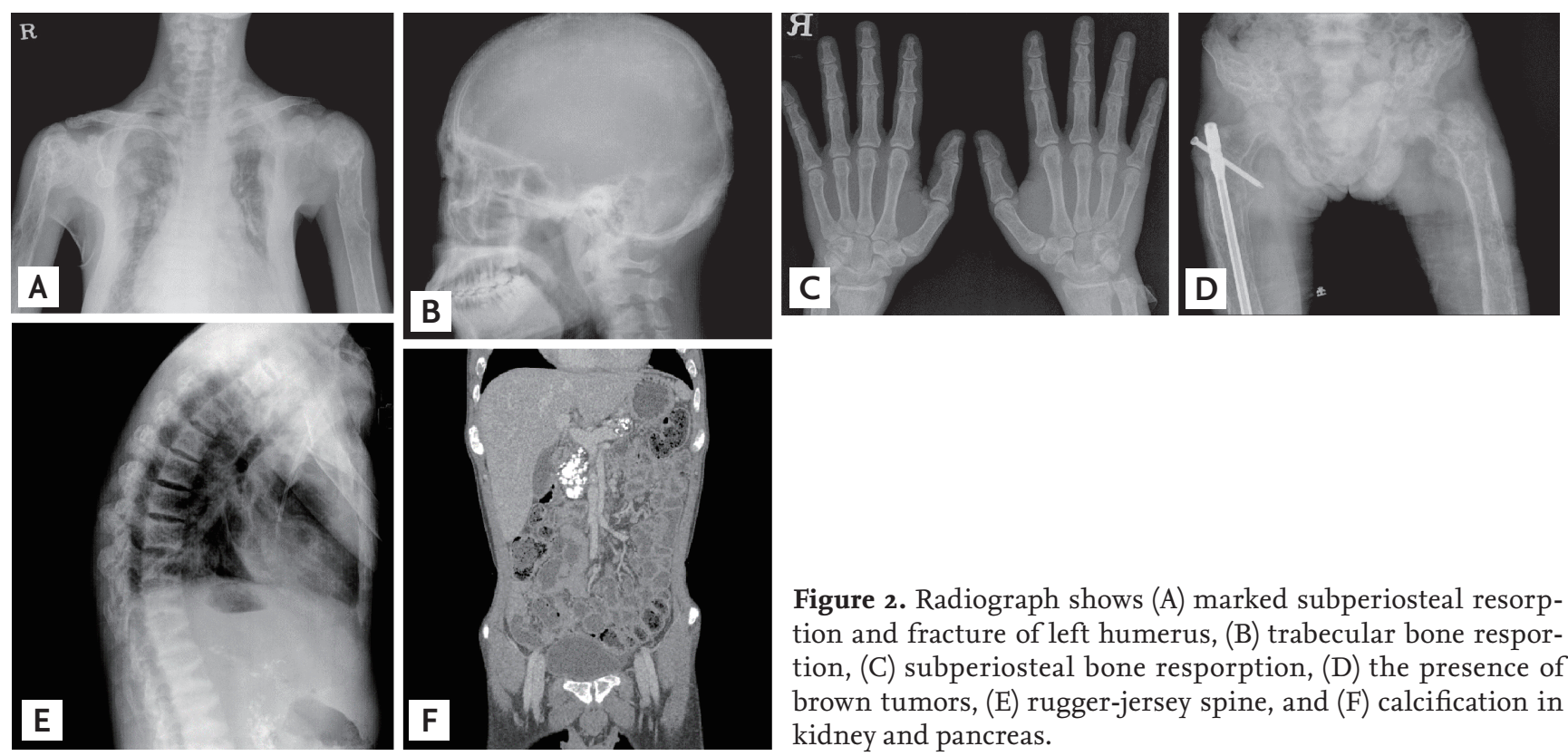

Figure 2. Radiograph shows (A) marked subperiosteal resorption and fracture of left humerus, (B) trabecular bone resportion, (C) subperiosteal bone resporption, (D) the presence of brown tumors, (E) rugger-jersey spine, and (F) calcification in kidney and pancreas. 
concentration 4,470 pg/mL (normal range, 9 to 55 ). The patient was treated with intravenous saline and zoledronic acid; however, his serum calcium concentration failed to return to normal levels. He was subsequently treated with cinacalcet, after which his serum calcium concentration steadily decreased to the normal range.

\section{Conflict of interest}

No potential conflict of interest relevant to this article was reported. 